\title{
Remote imaging by transfer of coherence functions through optical fibers
}

William Rhodes, Gisele Welch

William T. Rhodes, Gisele Welch, "Remote imaging by transfer of coherence functions through optical fibers," Proc. SPIE 10296, 1999 Euro-American Workshop Optoelectronic Information Processing: A Critical Review, 1029602 (2 June 1999); doi: 10.1117/12.365905

Event: Euro-American Workshop on Optoelectronic Information Processing, 1999, Colmar, France 


\title{
Remote imaging by transfer of coherence functions through optical fibers
}

\author{
William T. Rhodes*a and Gisele Welch ${ }^{\mathrm{b}}$ \\ ${ }^{\mathrm{a}}$ GTL-CNRS Telecom, UMR CNRS 6603, Georgia Tech Lorraine, \\ 2 rue Marconi, 57070 Metz, France \\ ${ }^{\mathrm{b}}$ Georgia Tech Research Institute, Georgia Institute of Technology, \\ Atlanta, Georgia 30332-0834, USA
}

\begin{abstract}
The spatio-temporal coherence function (STCF) associated with the wavefield from a scene conveys all information required to produce an image of that scene. Through the use of dynamic coding/decoding masks a STCF can be converted in form so as to allow the image-forming information to be conveyed via a single optical fiber to a remote viewing site. A general theory relating to STCF transfer is presented, and a Young's fringe-based discussion of why the scheme is plausible is presented.
\end{abstract}

\section{INTRODUCTION}

The holographic playback process is sometimes referred to as wavefront reconstruction. In this paper we describe a process that might be described as coherence-function reconstruction. The spatio-temporal coherence function associated with an optical wavefield does not contain all the information that the wavefield itself does; however, it contains all information required to produce a faithful image of a full three-dimensional, full spectral bandwidth scene, as viewed through a particular window. We describe a scheme whereby this information-loaded function of $x, y$, and $t$ can be coded in such a way that it can be transmitted via an optical fiber from a viewing site to a remote display.

The paper begins with estimates of the amount of information that can flow from an object through a "window" onto that object. The relevant spatio-temporal coherence function is then introduced, and the basic scheme for squeezing the associated information through a pinhole-and, as easily, through a fiber-is then presented. The importance of coherence functions in image formation is discussed, and a general theorem relating to coherence function transmittance is introduced. Finally, extensions to and implications for other areas of optics are briefly discussed.

\section{IMAGE TRANSMISSION INFORMATION REQUIREMENTS}

Before introducing the basic scheme, we look at the bandwidth requirements associated with different image-information transmission systems.

* Correspondence: Email: wrhodes@ee.gatech.edu; Tel. +33 387-20-3939; Fax +33 $387-$ 20-3940

1999 Euro-American Workshop Optoelectronic Information Processing: A Critical Review, edited by Philippe Refredier, Bahram Javidi, Proc. of SPIE Vol. 10296 (Vol. CR74), 1029602 (C) (1999) 2017 SPIE · CCC code: 0277-786X/17/\$18 - doi: 10.1117/12.365905 


\section{2 / Critical Reviews Vol. CR74}

\subsection{TV signals and EM field}

Conventional image transmission for remote display often involves a tremendous reduction in the information flowing from the scene. Color scenes, for example, are usually decomposed into three primary-color (e.g., RGB) 2-D images, as suggested in Fig. 1. If 3-D information is to be transmitted, an additional trio of RGB images might be transmitted to produce a color stereo pair.

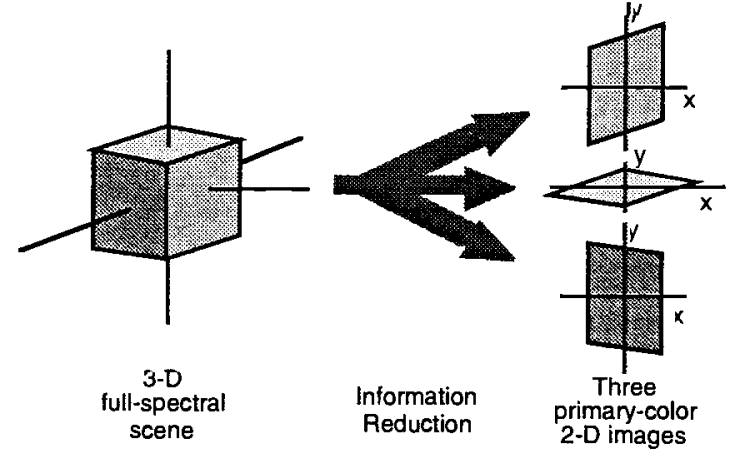

Fig. 1. Reduction of information in typical image transmission scheme: a full-color 3-D scene is reduced to three RGB primary-color images; two RGB trios are recorded for a stereo-pair.

The information reduction achieved is enormous. Consider, for example, the case illustrated in Fig. 2: A 3-D full-spectral-bandwidth (e.g., containing all colors in the $400-700 \mathrm{~nm}$ wavelength band) object is viewed through a $10 \mathrm{~cm} \times 10 \mathrm{~cm}$ window. We ask ourselves, how much information flows through that window in the form of electromagnetic wave "signal" information? Assuming that the mean wavelength of 550 $\mathrm{nm}$ gives the linear dimension of a spatial sample patch at the window, and assuming a spectral bandwidth of $3.2 \times 10^{14} \mathrm{~Hz}$, an amount corresponding to radiation in the 400-700 $\mathrm{nm}$ wavelength band, the number of Nyquist samples required per second to represent the information flowing through the window is of the order of $10^{23}$, a number far beyond the capabilities of the most advanced telecommunication system. In fact, to convey this information, a total of some $3 \times 10^{8}$ individual channels (e.g., fibers) would be required, each operating at a bandwidth of more than $10^{14} \mathrm{~Hz}$. An RGB stereo pair of TV images would typically require fewer than $10^{8}$ samples per second. However, the TV image does not provide a faithful reproduction of the actual spectral content of the scene, nor does it give full 3-D viewing: it only presents a stereo image from a single viewpoint.
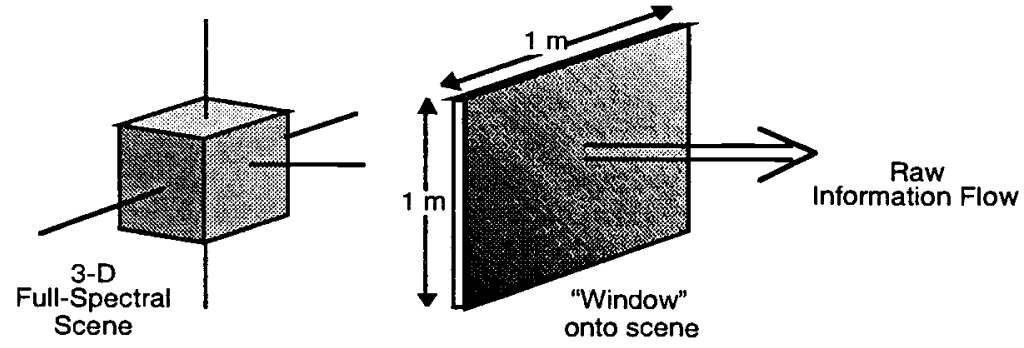

Fig. 2. The raw information conveyed by the optical electromagnetic field flowing through a $1 \mathrm{~m} \times 1 \mathrm{~m}$ window can be enormous. 


\subsection{Spatio-temporal coherence function}

We now make the following critical observation: In order to form a full threedimensional, full spectral-bandwidth image of the scene, it is not necessary to transmit all of the information associated with the electromagnetic field passing through the window. Rather, it is only necessary to transmit the greatly-reduced amount of information associated with a spatio-temporal coherence function of the field. We show in this paper that this spatial-temporal coherence function can be encoded in such a way that it can be transmitted to a remote receiver/display system via a single optical fiber.

We take the coherence function of concern to be the mutual coherence function $\Gamma_{12}(\tau)$ associated with the electromagnetic field (there are other functions that could be used as well in the analysis). It is defined, with the help of Fig. 3, by 1

$$
\Gamma_{12}(\tau)=\left\langle E\left(x_{1}, y_{1}, t+\tau\right) E^{*}\left(x_{2}, y_{2}, t\right)\right\rangle \text {, }
$$

where $\left\langle\cdot>\right.$ denotes a suitable time average, $E\left(x_{1}, y_{1}, t\right)$ is the complex electric field amplitude of the wave passing through a plane at point $\left(x_{1}, y_{1}\right)$, and $E\left(x_{2}, y_{2}, t\right)$ is that of the wave at a second point $\left(x_{2}, y_{2}\right)$. The mutual coherence function is all-important to the formation of an image. Indeed, if evaluated for an appropriate set of point pairs $\left(x_{1}, y_{1}\right)$, $\left(x_{2}, y_{2}\right), \Gamma_{12}(\tau)$ conveys all of the information that is needed to form an image of the object as viewed through the window. For planar incoherent objects, $\Gamma_{12}$ is a function of the vector separation between $\left(x_{1}, y_{1}\right)$ and $\left(x_{2}, y_{2}\right)$, and significant reduction in information content results. Because of the time average and the spatial sampling requirements for $\Gamma_{12}$, the actual information associated with the mutual coherence function can be enormously reduced in quantity from that associated with $E(x, y, t)$ itself and yet be fully sufficient for image formation. For example, allowing $\Gamma_{12}$ to have a temporal frequency bandwidth of $30 \mathrm{~Hz}$ (consistent with TV imagery) and to derive from a $10-\mathrm{cm}$ diameter, planar, $400-700 \mathrm{~nm}$ spatially-incoherent object at a distance of $1 \mathrm{~m}$, the information flow through the $10 \mathrm{~cm} \times 10 \mathrm{~cm}$ viewing window reduces to something of the order of $10^{10}$ samples per second.

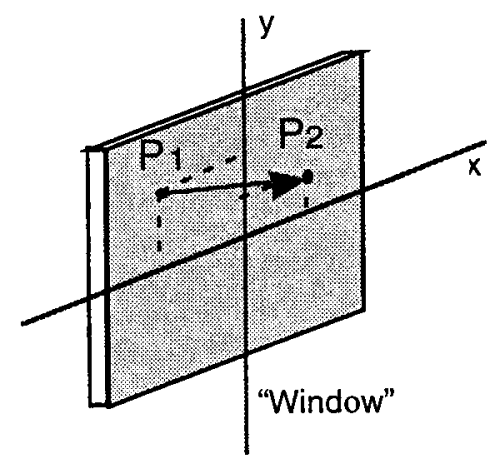

$$
\begin{aligned}
& \text { Mutual Coherence Function: } \\
& \Gamma_{12}(\tau)=\left\langle E\left(x_{1}, y_{1}, t+\tau\right) E^{*}\left(x_{2}, y_{2}, t\right)\right\rangle
\end{aligned}
$$

(.) Jenotes time average

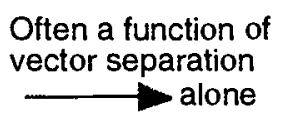

Fig. 3. Definition of the mutual coherence function $\Gamma_{12}$. 
4 / Critical Reviews Vol. CR74

\section{SQUEEZING THE INFORMATION THROUGH A PINHOLE}

Having noted the tremendous reduction in information that can result from going from the electromagnetic field passing through the window to the associated mutual coherence function, we now address a method for squeezing that information through a single pinhole. For simplicity, discussion is limited to the situation shown in Fig. 4: the object is not three-dimensional but, rather, planar, and it is assumed to be quasimonochromatic-i.e., single-color. Extension to the 3-D full-spectral-bandwidth case will be commented on later in the paper. Under the quasimonochromatic condition we need no longer work with the mutual coherence function but only with the mutual intensity, $J_{12}$, defined by

$$
J_{12}=\Gamma_{12}(0) \text {. }
$$

As suggested in the figure, the scheme requires the use of special dynamic masks, one of which can be viewed as performing a "coding" operation on the wavefield information the other performing a corresponding "decoding" operation. The system operates by means of a transfer, from input to output, of the mutual intensity function associated with the object wave. Incident on the coding mask is a wavefield with mutual intensity $J_{i n}$. Some of the light associated with the transmitted wavefield is squeezed, by system optics, so as to allow it to pass through the pinhole, whereupon it is expanded again to illuminate the decoding mask and to produce, on the output side, a mutual coherence function $J_{\text {out }}$ given by

$$
J_{\text {out }}=\beta J_{\text {in }}+\alpha J_{\text {scatter }},
$$

where $\alpha$ and $\beta$ are proportionality factors and where $J_{\text {scatter }}$ represents additional spatially incoherent scattered light.

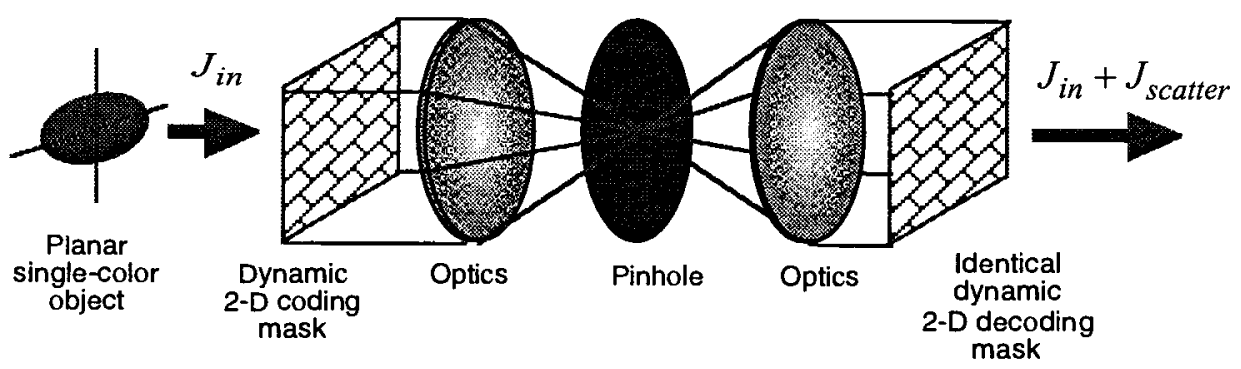

Fig. 4. Coherence function transfer through a pinhole. A dynamic 2-D "coding" mask converts the incident coherence information into a form that can be squeezed through the pinhole. An identical, and fully synchronized mask at the output end of the system reconstructs the coherence properties of the input wavefield. In addition to the coherence-restored component of the output wavefield, there is also a strong spatially incoherent component corresponding to conventional scatter light.

The way in which this coherence-transfer system can be used in the remote formation of an image is illustrated in Fig. 5. It is well known that the mutual intensity function associated with a wavefield is all that is necessary for the formation of a conventional image of the quasimonochromatic object producing the wave. ${ }^{2}$ Since the coherencetransfer system conveys to the output-to within an additive spatially incoherent scatterlight wave component-the coherence function of the light incident on the encoder mask, light from the decoder mask can itself be focused to the appropriate conjugate image plane 
1999 Euro-American Workshop on Optoelectronic Information Processing / 5

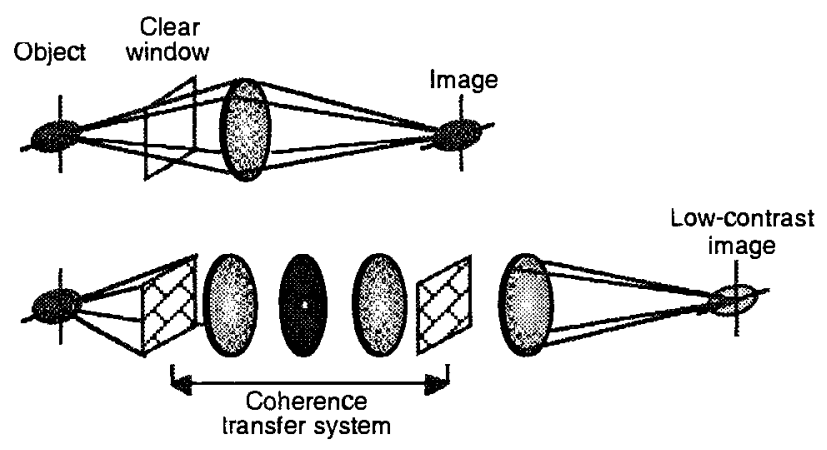

Fig. 5. Remote image formation via coherence transfer. Top: conventional image formation. Bottom: forming an image by imaging the coherence-restored component of the wavefield. The incoherent, or scatter-light component of the output of the decoder mask will typically reduce the contrast of the image quite seriously.

to form an image of the object. The presence of the incoherent scatter light reduces the contrast of the image-tremendously, in fact, for objects of large space-bandwidth product, to the point where the scheme is likely of little practical importance.

If the image is to be transmitted via an optical fiber, it is a simple matter to replace the pinhole with an optical fiber. The ultimate configuration then has the appearance shown in Fig. 6. The viewing station can be as distant from the input "port" as desired, subject to attenuation in the fiber. Dispersion does not affect performance; it only changes the time of arrival of different color components of the image. It is of course necessary that the fiber transmit the entire spectral bandwidth of the object if a full-color image is to be formed. The time required to form an image is the time required for the coding/decoding mask pair to execute a suitable "sequence" of states, a point discussed further below. In general this time can be as short as is allowed by the mask technology.
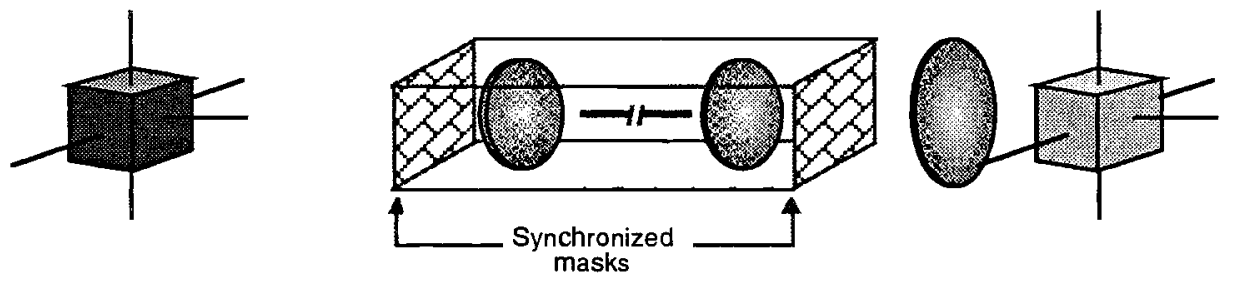

Fig. 6. Ultimate configuration, with an optical fiber replacing (and effectively extending longitudinally) the pinhole. The lens at the output of the coherence-transfer system forms a low-contrast image of the object viewed at the input end.

\section{COHERENCE FUNCTIONS AND IMAGE FORMATION}

We now take a moment to discuss in more detail the relation between the coherence function and image formation. The basic concept, well known to students of coherence theory, is presented with the help of Fig. 7. If a conventional optical imaging system is fitted with a pupil-plane mask containing two sufficiently small pinholes, the image of a 


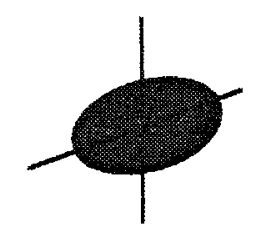

Object at wavelength $\lambda$

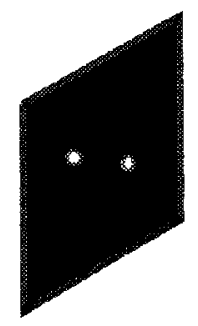

2-pinhole
mask

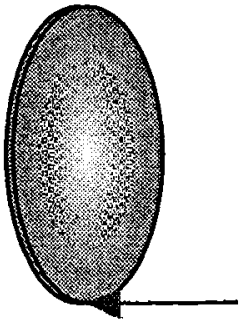

Image-forming lens

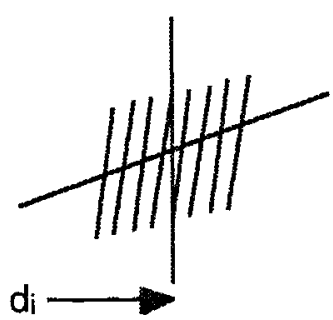

Sinusoidal fringes of spatial frequency $S / \lambda d i$

Fig. 7. The Young's fringes produced by an imaging system with a two-pinhole mask represent one Fourier component of the image intensity distribution.

quasimonochromatic object reduces to a sinusoidal fringe pattern-a set of Young's interference fringes - that conveys the magnitude and phase of a particular spatial frequency component of the image. ${ }^{3}$ By changing the vector separation of the two pinholes, it is possible, sequentially in time, to project on the image plane a succession of Fourier components of the image intensity distribution: in effect, to perform a Fourier synthesis of that image.

The operation of the coherence transfer system can be partially explained with the help of Fig. 8. The first part of the system illustrated is the same as that of Fig. 7: an imaging system with a two-pinhole mask produces a set of fringes in the image planeone spatial frequency component of the image intensity distribution. By means of a timevarying phase shifter, these fringes are made to move with constant velocity past the input end of the optical fiber. Light intensity at the fiber, given by

$$
I(t)=b+m \cos \left(2 \pi f_{o} t+\phi\right),
$$

conveys, through the parameters $m$ and $\phi$, the magnitude and the phase of the mutual intensity $J_{12}$ associated with the particular pinhole positions.

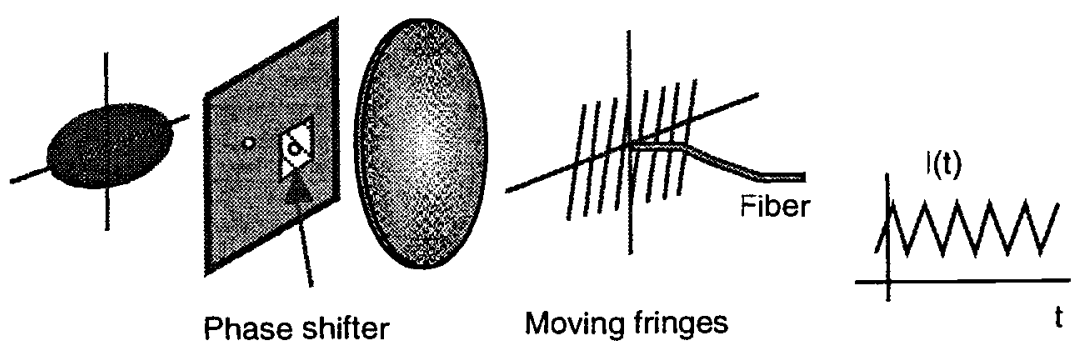

Fig. 8. Encoding the magnitude and phase of the mutual intensity by means of a phase shifter over one pinhole. Fringes move across the input end of the fiber, producing with time a biased sinusoidal intensity in the fiber whose magnitude and phase are governed by $\left|J_{12}\right|$ and $\arg \left(J_{12}\right)$. 
Decoding of the coherence information to produce the output image by time-sequential superposition of Fourier components is achieved through the use of an identical twopinhole mask with synchronized phase-shifter at the output end, as shown in Fig. 9. The two-pinhole mask produces, in the image plane, a set of moving fringes similar to those produced by the encoder system. As the fringes move, they vary in intensity because of the sinusoidally-varying intensity of the light emerging from the fiber. Through what is effectively a stroboscopic effect, the time-average fringe pattern has precisely the spatial phase of the measured $J_{12}$, and its contrast is proportional to $\left|J_{12}\right|$. In some sense, the more general coding mask in Figs. 4, 5, and 6 can be viewed as equipping the system with a multitude of pinhole pairs simultaneously, each pair, along with its decodingmask counterpart, conveying to the output of the coherence transfer system the magnitude and phase of $J_{12}$ appropriate for the positions of the pinholes.

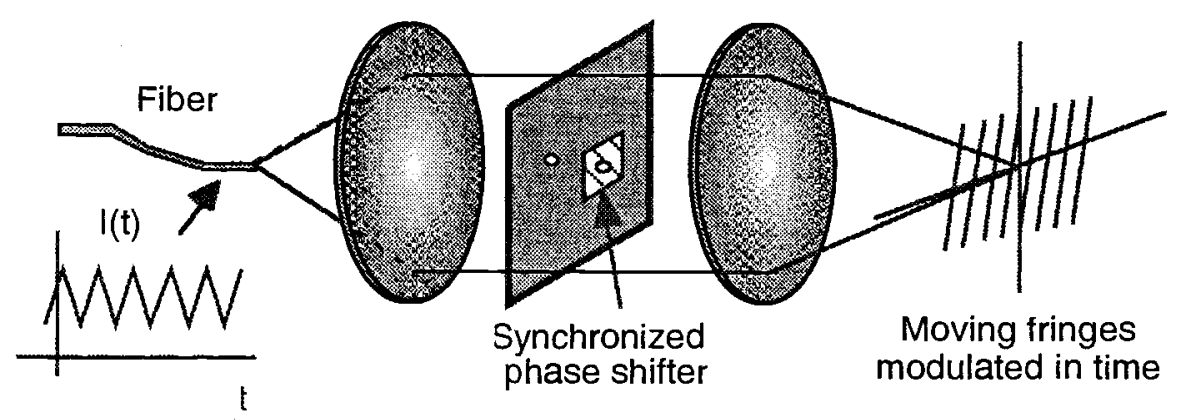

Fig. 9. Decoding of the coherence function. Moving fringes projected onto a conjugate image plane are "frozen" in the correct position by the stroboscopic effect of the sinusoidallymodulated intensity of the light conveyed by the fiber.

The ability of a suitable encoding/decoding mask pair to accomplish the feat suggested above is not immediately apparent. Support for the statement, in the form of a theorem, is presented in the following section. Assuming that the basic claim is valid, however, it still remains to extend it to cover the case of full-color, three-dimensional objects. That the scheme should also work with a full-spectral-bandwidth object follows directly from the nature of the Young's fringe formation with the two-pinhole system and from the linearity with wavelength of the image-forming process. Referring again to Fig. 7 , for a given pinhole pair, light of each color from the object produces a separate fringe pattern, each such pattern at its own wavelength $\lambda$, where the vector spatial frequency of the fringe structure is given by

$$
\vec{f}=\frac{\vec{s}}{\lambda d_{i}},
$$

$\vec{s}$ being the vector separation of the two pinholes and $d_{i}$ being the distance from the imaging lens to the image plane. Each wavelength is conveyed independently, and at the decoding end each color reproduces a Fourier component of the correct spatial frequency, i.e., consistent with Eq. (5). That the scheme will work with 3-D objects follows from the statement made earlier that the coherence function associated with the light incident on the encoder-i.e., on the "window" onto the object-is all that is necessary for the formation of an image, including a 3-D one. This fact, although often not considered among people working with image forming systems, is well-established. 


\section{COHERENCE TRANSFER THEOREM}

A basic theorem that underlies the claims asserted above is stated here, without proof. A derivation of the relationship will be presented elsewhere. ${ }^{4}$ Reference is made to Fig. $10(a)$, which shows a wave of complex amplitude $\mathbf{U}(x, t)$ incident on a dynamic random phase screen characterized by complex amplitude transmittance $\phi(x, t)$. After passage through the screen, the wave passes through a system that is linear in wave amplitude, to pass subsequently through an identical dynamic random phase screen (it is not essential that the screen be a pure phase element), to yield output wave $\mathbf{U}^{\prime}(x, t)$. The same system is illustrated in block-diagram form in Fig. 10(b). The coherence transfer theorem has the following form:

$$
J_{o u t}\left(x_{1}, x_{2}\right)=a\left(x_{1}\right) \delta\left(x_{1}-x_{2}\right)+b\left(x_{1}, x_{2}\right) J_{\text {in }}^{*}\left(x_{1}, x_{2}\right),
$$

where $J_{\text {out }}$ is the mutual intensity of the output wave $U^{\prime}(x, t), J_{\text {in }}$ that of the input wave $U(x, t)$, and where $a\left(x_{1}\right)$ and $b\left(x_{1}, x_{2}\right)$ are given by

$$
\begin{gathered}
a\left(x_{1}\right)=\gamma^{2} \int_{-\infty}^{\infty} I(\xi)\left|h\left(x_{1}, x_{2}\right)\right|^{2} d \xi \\
b\left(x_{1}, x_{2}\right)=\gamma^{2} h\left(x_{1}, x_{2}\right) h^{*}\left(x_{2}, x_{1}\right) .
\end{gathered}
$$

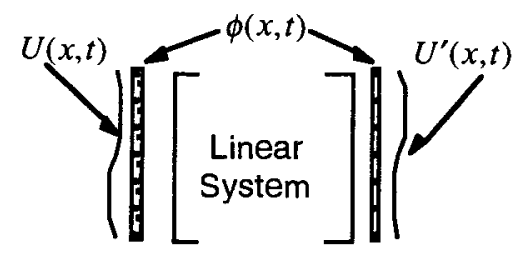

(a)

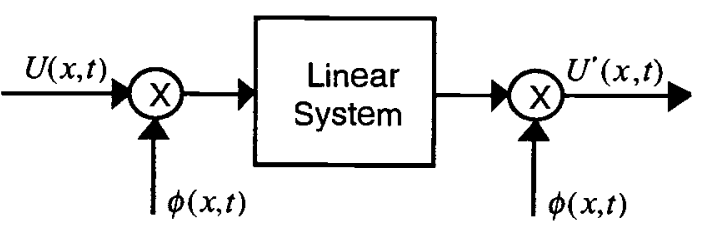

(b)

Fig. 10. Double passage system: (a) quasi-physical model; (b) schematic representation. $U(x, t)$ and $U^{\prime}(x, t)$ denote the complex amplitudes of quasimonochromatic input and output waves, and $f(x, t)$ denotes the complex transmittance of the scattering screen. Examples of the interviening linear system include free-space propagation, imaging by a lens, and transmission through a photomask.

In these latter expressions $h(x, \xi)$ is the impulse response associated with the linear system, $I(x)$ is the optical intensity $\left\langle|U(x, t)|^{2}\right\rangle$ of the incident wave, and $\gamma$ is a proportionality constant. In deriving equation (6) it is assumed that the random phase screen is "good" in the sense that (a) the phase excursions are large compared to $2 \pi$ (if the screen were used to produce laser speckle, the speckle would be declared to be "fully developed"), and (b) the screen-which can be visualized as a moving diffuser-is deltacorrelated, meaning that the scattering structure is small compared to any other scale of concern in the problem.

With reference to Fig. 4, the "system" represented by $h(x, \xi)$ is in the case of interest to us an imaging system with a pinhole aperture, and $h(x, \xi)$ thus equals (for a sufficiently small pinhole) a constant - the imaging blur function is much larger than any detail in the image. The function $b\left(x_{1}, x_{2}\right)$ is, in turn, through Eq. (8), constant, and Eq. (6) assumes the form 


$$
J_{\text {out }}\left(x_{1}, x_{2}\right)=a_{o} \delta\left(x_{1}-x_{2}\right)+b_{o} J_{\text {in }}^{*}\left(x_{1}, x_{2}\right),
$$

$a_{\mathrm{o}}$ and $b_{\mathrm{O}}$ constants. The two terms in this expression represent two different contributions to the output wave $U^{\prime}(x, t)$. The first term corresponds to a fully incoherent contribution, a contribution that produces only a bias, or background "glow," to any image that one might attempt to form with the doubly-scattered light. The second term, on the other hand, has, to within an inconsequential conjugation, exactly the same coherence properties as the wave incident on the first scattering screen. This wave contribution can be used to form an image of any object that can be "seen" by the first screen.

\section{CONCLUDING REMARKS}

A detailed analysis of the ramifications of the coherence function transfer theorem presented above has yet to be conducted. We know, however, that this theorem can be used to explain not only the "image-through-an-optical-fiber" scheme presented above but also (a) the functioning of an entire class of super-resolving image forming systems first described by Lukosz $z^{5}$ and (b) the counter-intuitive double-passage phenomenon known as enhanced backscatter ${ }^{6}$. Indeed, our initial exposure to the coherence-function transfer concept came via a study of enhanced backscatter and Lukosz-type systems.

For us perhaps the most significant consequence of our preliminary studies in this area has been an enhanced appreciation of the fundamental importance in image formation of spatio-temporal coherence functions associated with a wavefield. Several texts show how, e.g., the mutual intensity function can be used in the analysis of an image forming system, but these texts do not convey clearly to the reader the fact that these STCFs are the information-bearing quantities of concern. We believe that an analysis based in STCF transfer can be used to extend considerably the understanding of Lukosz-type systems, and we suspect that a good understanding of STCF transfer systems could lead to information processing applications.

\section{REFERENCES}

1. A. S.. Marathay, Elements of Optical Coherence Theory, (John Wiley \& Sons, New York, 1982).

2. Joseph Goodman, Statistical Optics, (John Wiley, New York, 1985), Chap. 5.

3. This statement is strictly true only for the case of a spatially incoherent, quasimonochromatic object.

4. William T. Rhodes and Gisele Welch, "Transfer of coherence functions by double-passage through dynamic diffracting screens," in preparation.

5. W. Lukosz, "Optical systems with resolving powers exceeding the classical limit," J. Opt. Soc. Am. 56, 1463-1472 (1966), and 57, 932-941 (1967).

6. A.G. Vinogradov, Yu. A. Kravtsov, and V.I. Tatarskii, "The effect of intensification of back-scattering by bodies that are situated in a medium having random inhomogeneities," Radiophys. Quantum Electron. 16, 818-823 (1973).

7. Gisele Welch, Application of coherence theory to enhanced backscatter and superresolving optical imaging systems," Ph.D. thesis, Georgia Institute of Technology, September 1995. 